\title{
Quality check protocol for control the losses of power on large associations of photovoltaic generators
}

\author{
A. López Agüera ${ }^{1}$, I. Fernández Otero ${ }^{1}$, R. Martínez Farreres ${ }^{2,1}$ and I. Rodríguez Cabo ${ }^{1}$ \\ ${ }^{1}$ Department of Particle Physics \& Galician Institute of High Energy Physics \\ Astroparticle Group. Santiago de Compostela University \\ South Campus 15702 Santiago de Compostela (Spain) \\ phone:+34 981 563100, fax:+34 981521091, e-mail: lopez@fpaxp1.usc.es, \\ ${ }^{2}$ Astroparticle and Special Plasmas Group. Alcalá de Henares University. \\ Mailing address rakelmarfe@hotmail.com
}

\begin{abstract}
The economic balance of large area photovoltaic generators suffer from even small losses on efficiency. The present work studies the causes of losses of power causes by the solar panels: The rigor of the manufacturer fullfiling the generators specifications, the dispersion on the electric characteristics of the generators associated either in series or in parallel, and not less important, the effect of the working temperature on the effective power of the generator. Modules quality, uniformity and time stability have been tested. The final results give rise to some recommendations to optimize the efficiency of large association of generators. The validity of those recommendations is discussed with reference to a sample 5

of 10 experimental data collected in the grid of $400 \mathrm{PV}$ from the Pierre Auger Project.
\end{abstract}

\section{Keywords:}

Photovoltaic system, Solar panels. Efficiency. Associations. Uniformity, Remote Control.

\section{Introduction}

The first applications of the photovoltaic generators were low power installations covering small areas. Nowadays, the situation is drastically changed and the photovoltaic installations became more and more powerful and the generators cover large areas. In the actual conditions, to ensure a controlled performance is crucial to profit of a positive economic balance. It is for this reason that remote control systems are being developed to optimize the final performance. Also the rigor of both manufacturer and installation need to be careful checked.

Main causes of losses on power are related to module production quality, the dispersion on the association of the cells and the working conditions. A efficient remote control will follow the PV system both before installation and during the normal working operation.

In the following, the procedures performed for the different tests as well as the obtained results are described. The full work has been developed over experimental data samples.
The experimental set-up used is the PV power systems of the Pierre Auger Observatory, PAO [1]. This observatory is designed as a grid of 1600 isolated stations. All these stations are distributed over an area of $60 \times 60 \mathrm{~km}^{2}$ located in the Malargüe high plateau in Argentina. Each station, designed to be an isolated self-power system, is being continuously monitored by means of a data logger and a radio link. The statistic collected along a year is around $10^{8}$

\section{2.- Photovoltaic system description}

The photovoltaic system has been designed to provide the needed power of the Auger Surface Detector (SD) stations, designed to be below $240 \mathrm{Wh}$ per day. It supplies power for the electronic read-out and the communication radio link. The system, formed by two solar panels serial connected and two regulated $\mathrm{Pb}$-acid batteries is sketched in Figure 1. According to the theoretical calculations, $100 \mathrm{~W}$ is required per each photovoltaic system to fulfill the power needs [1].

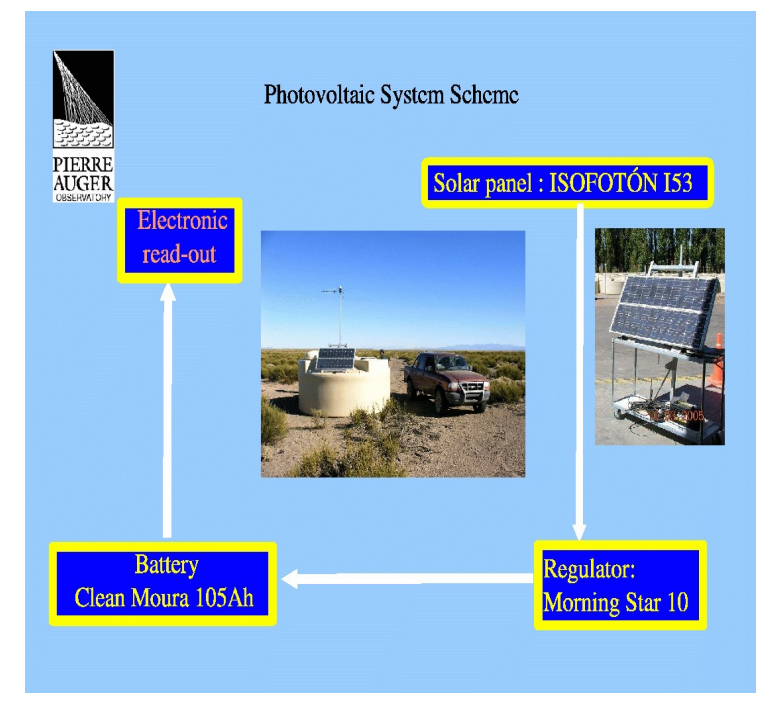

Fig 1: Photovoltaic system scheme with photos of the main components 
The panels acquired are the ISOFOTON model I-53/12V [2](see Table I for the electric characteristics).

\begin{tabular}{|l|c|}
\hline Isc (Sort circuit current) & $3.27 \mathrm{~A}$ \\
\hline Voc (Open circuit Voltage) & $21.6 \mathrm{~V}$ \\
\hline Imax (Maximum current) & $3.05 \mathrm{~A}$ \\
\hline Vmax (Voltage at Imax) & $17.4 \mathrm{~V}$ \\
\hline
\end{tabular}

Table I: Electric characteristics of the ISOFOTON W53 photovoltaic module for standard conditions

$$
\left(1000 \mathrm{~W} / \mathrm{m}^{2}, 25^{\mathrm{O}} \mathrm{C}\right) \text {. }
$$

Figure 2 shows the I-V characteristic curve expected from a crude simulation of the panel equivalent circuit which reproduces the specifications given by the manufacturer. Main panel characteristic parameters are marked. The working current, Iwork, at the working voltage , Vwork, can be approximated using a linear interpolation :

$$
I_{\text {work }}=\left(I_{S C}-\frac{I_{S C}-I_{\max }}{V_{\max }} V_{\text {work }}\right) F\left(V_{\text {work }}\right)
$$

where Imax y Vmax are the panel current and the voltage values at the point of maximum power and $\mathrm{F}$ is a correction factor to account for the curvature of the characteristic I-V curve in the region around the maximum power (see figure 2). We use the values 1.05 as suggested in previous studies (IES private communication) for a Vwork of $15 \mathrm{~V}$.

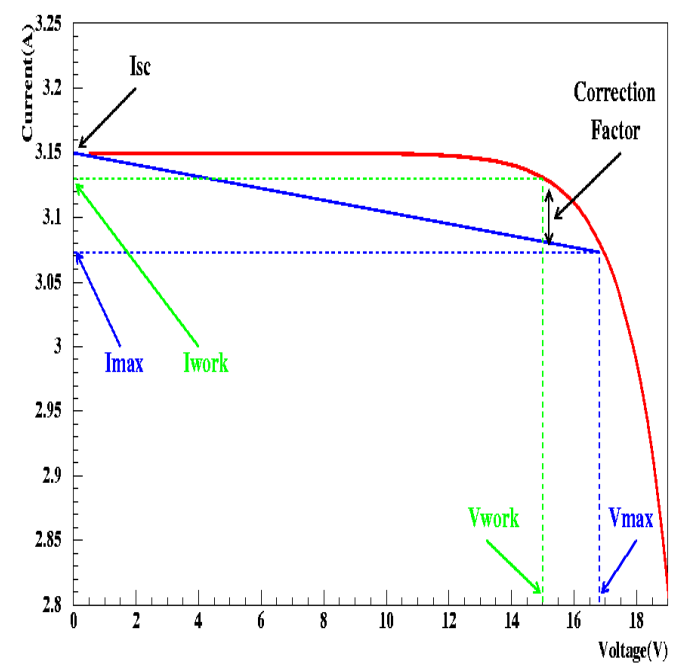

Fig. 2: Characteristic I-V curve for the I-53 photovoltaic modules

The power obtained from the panel mainly depends on the photovoltaic cell temperature, and the received radiance. Figure $2 \mathrm{~b}$ shows the simulated $\mathrm{I}-\mathrm{V}$ curve at different radiance values. The geometrical orientation of the panel affects the radiance collection. To ensure maximum power in winter, the panels are oriented to the North and tilted 55 degrees.

\section{Losses on efficiency causes by the dispersion on the production parameters : Uniformity}

The measurement of the static characteristics for each panel, such as short circuit current, the open circuit voltage or the current and voltage at maximum power should give a rather precise idea of the quality of the solar cells.

The purchase conditions were a working power above 50 $\mathrm{W}$ and a working current higher than $3.15 \mathrm{~A}$. A production quality check is required to the manufacturer (flash data). The accepted tolerance is 5\% (see Figure 3). Directly from the manufacturer data, $5.10 \%$ of the panels are discarded.

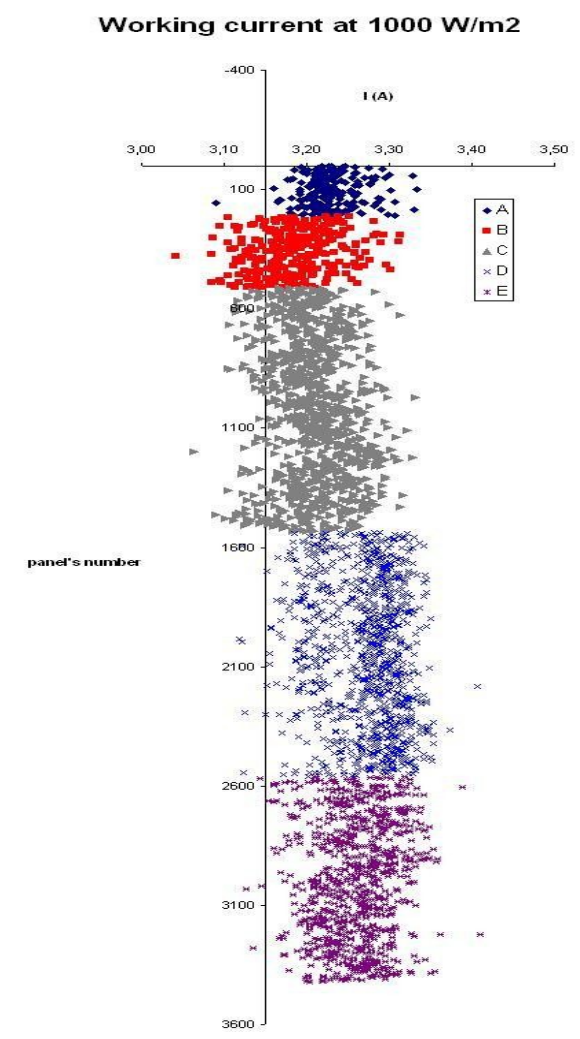

Fig. 3. Working Current for each panel arbitrary numerated. Different colors correspond different purchases.

To cross-check the manufacturer information in real conditions, we perform a simple quality check procedure. The test has been done over a sample of 53 panels taken randomly. The quality of a given panel is evaluated by a direct comparison with a calibrated panel used for reference [3]. As both panels are working in the same conditions, dependence on the radiance level is neglected. Moreover, modules are pre-heated during at least one hour in advance to avoid temperature dependence. During the testing procedure, panels are oriented with the same tilt angle. 
The experimental set-up is shown in figure 4 . The voltage between the shunt born measured for both under test, $\mathrm{V}_{\mathrm{sh}}{ }^{\text {test }}$, and calibrated, $\mathrm{V}_{\mathrm{sh}}^{\text {calib }}$, panels are a direct measurement of the short circuit currents. The use of a precision shunt resistor ( $1 \%$ tolerance) to measure working currents minimizes the uncertainties. For statistics, five measurements were done within 5 minutes (one per minute). The mean value and the quadratic estimated error are obtained.

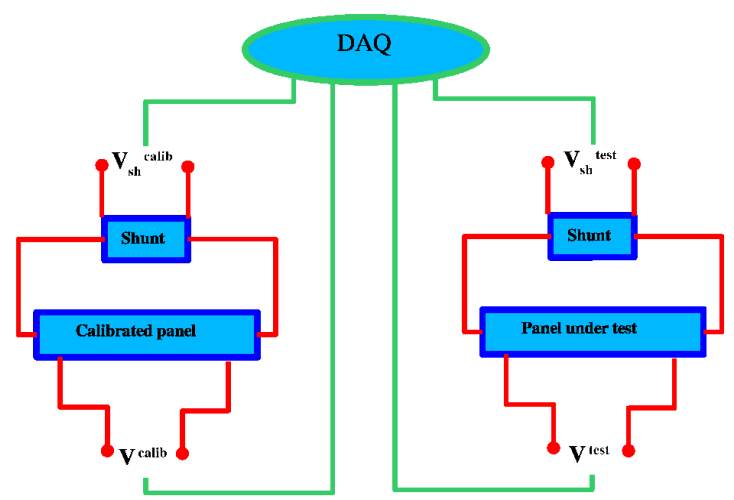

Figure 4: Electrical Scheme for direct test.

We have compared our direct test with the static measurement provided by the manufacturer. Figure 5 shows the ratio between the short circuit current from our test make in working conditions and the static flash given by ISOFOTON. The ratio is compatible with one within the experimental tolerance quoted above. Error bars can be limited to $2 \%$ due to the uncertainty in the shunts. The response is linear and the slope is close to one as expected.

The good agreement obtained between both nominal and measured generator characteristic, allows us to conclude that the manufacturer test is reliable and is sufficient to estimate the quality of the panel.

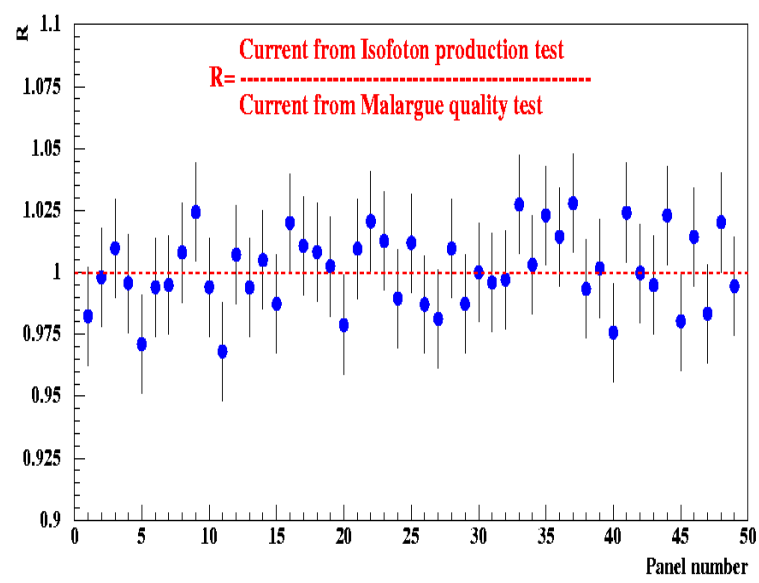

Fig. 5. The ratio between manufacturer and our own measurements for the short circuit current of 53 panels as a function of an arbitrary panel number. The error bars take into account the experimental error in the denominator.

\section{Losses on efficiency causes by the dispersion on the cells performance}

The electric characteristic of the generators always shows some dispersion. The association of different generators forming a large grid of generators, suffer from the performance of the worst module. The complete installation working conditions are far from the nominal optimal conditions. In the following the losses associated to the cells dispersion are quantified.

To decrease as much as possible the dispersion and since the panels are serial connected, it is essential that each pair have similar electrical behavior, i.e. similar short circuit current, to optimized the performance of the system. For a quick identification we require to the manufacturer to flag the generators corresponding to high, medium and low values of the short circuit current.

In we consider the full PAO as a sample of 1600 photovoltaic associations, we select two subsample: One formed with the lower values short circuit current (sample 1) and other with the highest sort circuit values (sample 2). The sensitive variables used in the analysis are the battery State of Charge (SoC) and the integrated frequency on regulation regime.

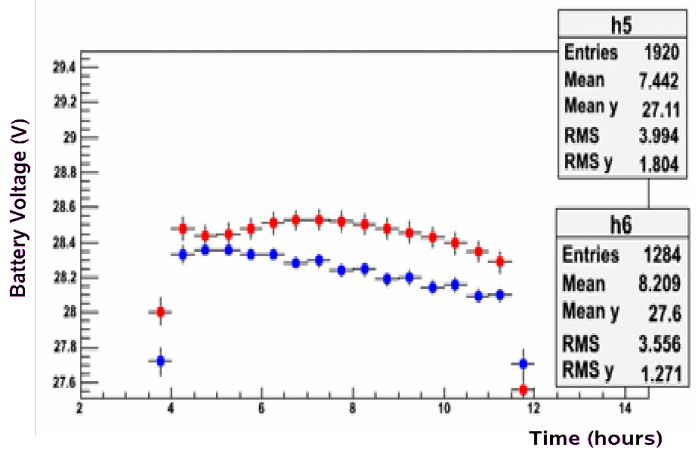

Fig. 6 . Voltage distribution shape along the day. In blue sample 1 . In red sample 2.

As example, Figure 6 shows the battery voltage mean value along a day. The sample 2 (in red) presents, as expected, always a high SoC than the sample 1 (in blue).

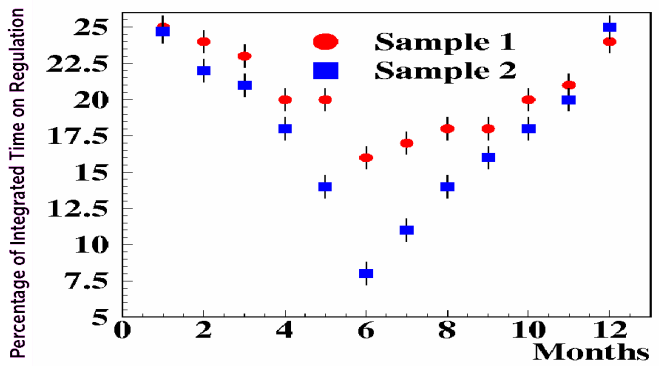

Fig. 7. Monthly percentage of integrated time on regulation regime. 
The same comparison in terms of the integrated frequency (see figure 7) on regulation regime give differences between samples in winter close to $20 \%$.

If the efficiency losses are measured evaluating the probability along the year to have the batteries completely charged (working on regulation regime), differences between $4 \%$ and $10 \%$ are observed depending on the association quality.

\section{Losses on efficiency causes by the working conditions.}

Once installed in the field the a photovoltaic system must continuously work during the 20 years of expected lifetime of the installation. To ensure a correct behavior, some stability criteria must be fixed. In the case of the solar panels, we will have to control working voltage using the monitoring data [4].

Figure 8 shows the monthly mean value of the voltage for the two serial connected panels in the station. The differences observed between winter and summer correspond to the radiance/temperature variation and can not be avoid.

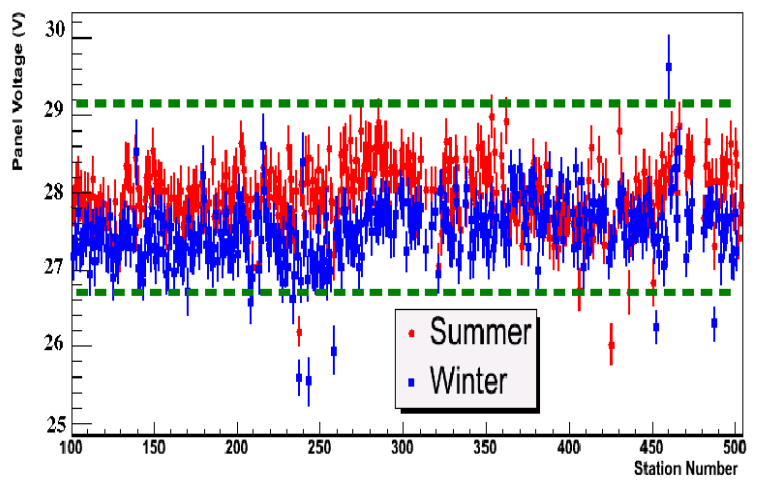

Fig. 8. Monthly mean Panel Voltage in charge regime vs an arbitrary number of station.

Unfortunately, the working current depends on the batteries working regime (charge, discharge or regulation). Because batteries are in series with the panels, the working current of the panel (see figure 9) can only be measured during the charge regime independently from the rest of the system. In these conditions we can only rely on the working current stability but not on the actual values.

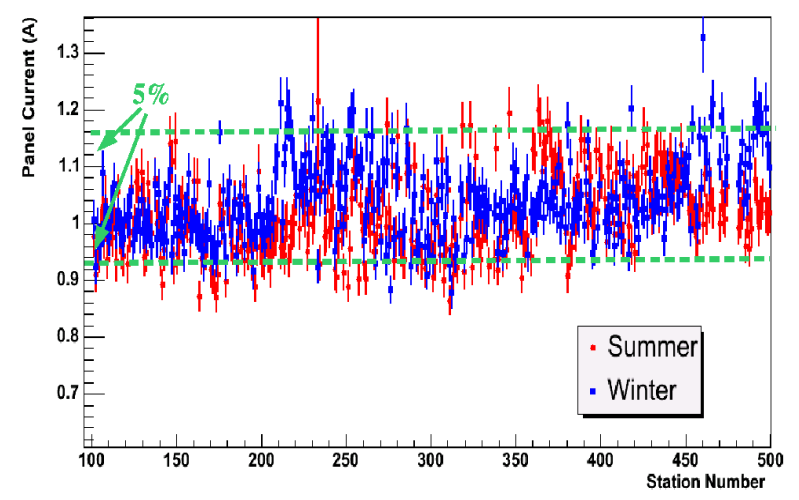

Fig. 9. Monthly mean Panel Current in charge regime vs an arbitrary number of station.
As expected, the sample spread is well within the 5\% tolerance measured during the static tests.

Both figures, 8 and 9, show information for the first 400 installed stations. Failures, directly related to the panels, have not been detected for the near 36 months of operation as it should be expected due to the panels robustness.

Besides, the hard meteorological conditions (wind blast, hail and large temperature oscillations) will allow studies of resistance of panels in extreme working conditions.

\section{Conclusions and recommendations.}

The complete set of 3420 ISOFOTON I53 solar panels of the PAO has been tested. Manufacturer uniformity fulfill the required technical parameters. The dispersion on the panel parameters can be evaluated in about $10 \%$ in the basis of a 5\% tolerance of the purchase requirement.

Since the panels are serial connected, it is essential that each pair have similar electrical behavior, i.e. similar short circuit current, to optimized the performance of the system.

Once installed, to ensure a correct behavior of the photovoltaic system, we perform working voltages stability tests. The PV is stable within a $8 \%$ tolerance .

The uniformity and stability tests performed constitutes a powerful tool to study the panel resistance in adverses meteorological conditions as well as to develop aging evaluation methods which have never being before.

No failures directly related with the solar panels has been detected for the first 400 stations installed.

\section{Acknowledgments}

The authors are grateful to the full technician team of the Southern Auger Observatory and the Isofoton team. Moreover to the PROFIT project (by means of Isofotón): 5050-XS25-64300 which partially support the job.

\section{References}

[1] "The Pierre Auger Project" in Nucl. Instrum. Meth A523 pp 50-95, 2004.

[2] www.ISOFOTON.org

[3] A. López-Agüera, E. Lorenzo, E. Marqués, G. Parente, I. Rodriguez-Cabo, E. Zas. Solar Panels for Auger Southern Observatory: A quality check. GAP2005-106.

[4] I. Rodríguez Cabo. Graduation Thesis. 